\title{
Convergence of Numbers of Synapses and Quantum Foci Within Human Brain Space: Quantitative Implications of the Photon as the Source of Cognition
}

\author{
Michael A. Persinger \\ Laurentian University, Sudbury, P3E 2C6, Ontario, Canada \\ E-mail address: mpersinger@laurentian.ca
}

\begin{abstract}
Quantitative solutions involving classical methods indicated that the numbers of quantum foci within the human cerebrum are the same order of magnitude as the numbers of synapses in the cerebral cortices. The Bohr frequency for the magnitude of the quantum associated with the removal of one nucleus from another is within the range of the width of synapse $(\sim 1 \mu \mathrm{m})$ while the recoil energy from a rest mass photon is equivalent to the energy produced by the entire field of dynamic neurons per second. The intrinsic energy $\left(10^{-20} \mathrm{~J}\right)$ associated with each action potential is similar to the gravitational potential energy from Planck's mass applied across $1 \mu \mathrm{m}$. Both the endogenous cerebral magnetic field strength which is similar in magnitude to intergalactic intensities and the interaction between weak geomagnetic variations and the cerebrum's electric dipole current are associated with photon flux densities in the order $\left(10^{-11} \mathrm{~W} \cdot \mathrm{m}^{-2}\right)$ that have been measured in the laboratory. The perspective of the human cerebral volume as a field of foci of photon quanta offers different perspectives for the intrinsic nature of consciousness and cognition and their influence by phenomena from astronomical origins.
\end{abstract}

Keywords: photons; synapses; Poynting Vector; neuroquantum; recoil energies; Bohr nucleus substitution frequency; consciousness-light field; gravitational-photon equivalence

\section{INTRODUCTION}

The transformation from the emphasis upon force to energy and the realization that the equivalence between energy and matter could be quantified during the late $19^{\text {th }}$ century and early $20^{\text {th }}$ century is now initiating a shift in scientific paradigms within chemistry and biology. For every aggregate of matter there should be a parallel energetic manifestation. The possibility that all forms of energy are interactive within space and time, one of the basic premises for arguments for "excess correlations" or "entanglement" [1,2], suggests a quantitative equivalence based upon contemporary values for fundamental physical and cosmological constants between foci of space and energy within the human brain should exist.

From this perspective the similarity for the time $\left(\sim 10^{-16} \mathrm{~s}\right)$ required for a photon moving at $\mathrm{c}$, the velocity of light in a vacuum $\left(3 \cdot 10^{8} \mathrm{~m} \cdot \mathrm{s}^{-1}\right)$, through a plasma cell membrane of $\sim 10^{-8}$ $\mathrm{m}$ width and the time required for a single orbit around the Bohr magneton may not be 
coincidence. The convergence may be a condition for which information from photons originating from anywhere could affect plasma membrane potentials or they could affect subtle properties of emerging photons.

The shift from information as Shannon binary codes to Gabor-like wavelets allows consideration of quantum holographic vector fields [3]. However, quantitative convergences between the known features of brain space and photon properties are required. In this paper, the role of recoil energy from photons and atoms and the gravitational potential within synaptic space are related to the solution that the numbers of synapses within the human brain are the same order of magnitude as numbers of quanta.

\section{NUMBER OF QUANTA WITHIN THE CERERBAL VOLUME}

Around 1908 J. J. Thompson employed the imaginary "photon cylinder" as a method to infer the numbers of quanta associated with photon densities. The discrete solutions were considered verifications that the energy was contained within heterogeneous discrete loci rather than homogeneously distributed. This conclusion was obtained by assuming the volume of the photon cylinder ( $\pi \mathrm{r}^{2}$ of a small space) for a given photon's wavelength multiplied by $3 \cdot 10^{8} \mathrm{~m}$. By dividing the resulting energy density by the energy per quantum the numbers of quanta per volume could be estimated [4].

The basic quantum unit of neural activity associated with the action potential and presumably with more complex biological processes such as "thinking" and "consciousness" can be calculated by the effect of $1.3 \cdot 10^{-1} \mathrm{~V}$ upon each unit charge $\left(1.6 \cdot 10^{-19} \mathrm{~A} \cdot \mathrm{s}\right)$ or $2.1 \cdot 10^{-20} \mathrm{~J}$ $[5,6]$. The basic space within which this energy is ultimately applied is the synapse. Although the two interfaces are separated by only $\sim 10^{-8} \mathrm{~m}$, the widths of the faces vary between $0.5 \mu \mathrm{m}$ and $2 \mu \mathrm{m}$ [7]. Assuming an average of $1 \mu \mathrm{m}$ for the width of a synapse, the surface area would be $10^{-12} \mathrm{~m}^{2}$. The resulting volume from the photon cylinder of $2.36 \cdot 10^{-4} \mathrm{~m}^{3}$ indicates that the energy density would be $0.89 \cdot 10^{-16} \mathrm{~J} \cdot \mathrm{m}^{-3}$.

The energy per quantum for cerebral activity should reflect the fundamental frequency. Considering the peak spectral power density that occurs around $10 \mathrm{~Hz}$, the energy would be $6.63 \cdot 10^{-33} \mathrm{~J}$ per quantum. As a result the number of quantum per volume is $0.13 \cdot 10^{17}$ quantum per $\mathrm{m}^{3}$. Because the average volume of the human cerebrum is $1350 \mathrm{cc}(\mathrm{SD}=176 \mathrm{cc})$ or $1.35 \cdot 10^{-3} \mathrm{~m}^{3}$, the numbers of quantum per cerebrum would be $\sim 1.8 \cdot 10^{13}$. This is well within the measurement error for the estimated numbers of synapses within the human cerebral cortices [7]. The congruence suggests that from a perspective of quantum biology, the energy within the punctate space of a synapse is a functional quantum.

\section{THE POYNTING VECTOR}

The Poynting Vector can be inferred to discern the direction the energy is moving within space-time. The classical representation is:

$$
\mathrm{S}=\mathrm{u}_{\mathrm{o}}^{-1}\left[\mathrm{~V} \cdot \mathrm{m}^{-1}\right] \mathbf{x} \mathrm{B}
$$

where $\mu_{\mathrm{o}}=4 \pi \cdot 10^{-7}$ which results in $\mathrm{kg} \cdot \mathrm{s}^{-3}$ or $\mathrm{W} \cdot \mathrm{m}^{-2}$. 
Assuming an average of $3 \mu \mathrm{V}\left(3 \cdot 10^{-6} \mathrm{~V}\right)$ between the rostral-caudal portion of the cerebrum $\left(10^{-1} \mathrm{~m}\right)$, inverse $\mu=8 \cdot 10^{5}$ and the intrinsic cerebral magnetic fields strength of $2 \cdot 10^{-12} \mathrm{~T}$, the radiant flux density would be $\sim 5 \cdot 10^{-11} \mathrm{~W} \cdot \mathrm{m}^{-2}$.

This quantity is within the range, as measured by photomultiplier tubes, of the background levels within very dark conditions sufficient to measure variations in photon emissions from both cell cultures [8] and human brain activity [9].

The product of magnetic field strength $\left(\mathrm{kg} \cdot \mathrm{A}^{-1} \cdot \mathrm{s}^{-2}\right)$, charge $(\mathrm{A} \cdot \mathrm{s})$ and frequency-squared $\left(\mathrm{s}^{-2}\right)$ is $\mathrm{kg} \cdot \mathrm{s}^{-3}$. The operating magnetic field strength of the cerebral magnetic fields $\left(2 \cdot 10^{-12} \mathrm{~T}\right)$, the unit charge $\left(1.6 \cdot 10^{-19} \mathrm{~A} \cdot \mathrm{s}\right)$ and the square of the neutral hydrogen frequency $\left(1.42 \cdot 10^{9} \mathrm{~Hz}\right)$ results in $0.64 \cdot 10^{-11} \mathrm{~W} \cdot \mathrm{m}^{-2}$. This is also within the specific range of power densities associated with photon emissions from the cerebrum when a person imagines white light [10].

The energy associated with such photon emissions has been shown to equivalent to the magnetic energy associated with the simultaneous decrease in intensity of the earth's magnetic field up to $\sim 1 \mathrm{~m}$ of the person's cerebrum [11]. The measured values of decreases in proximal static geomagnetic intensity as the cerebral photon intensity increased were $15 \mathrm{nT}$ and $5 \mathrm{nT}$ at distance of $0.25 \mathrm{~m}$ and $1 \mathrm{~m}$, respectively.

Both the calculated magnetic energy decreases from the earth's magnetic field and increases from the photon emissions were $\sim 10^{-11} \mathrm{~J}$. This is equivalent to about 100 millions neurons discharging at $10 \mathrm{~Hz}$.

\section{INFLUENCE OF AMBIENT GEOMAGNETIC FIELDS}

Vares and Persinger [12] found an inverse correlation between ambient geomagnetic intensity and background photon emission density from the same space. For approximately every $1 \mathrm{nT}$ increase in geomagnetic intensity there was a $10^{-12} \mathrm{~W} \cdot \mathrm{m}^{-2}$ decrease in photon density. Hunter et al [11] as well as Saroka et al [13] had found similar inverse correlations between the shift in the geomagnetic field strength around the heads of individuals and the density of photon emissions.

Recently Persinger et al [14] measured discrete changes in cerebral photon emissions that strongly supported the hypothesis of $\mathrm{Wu}$ and $\mathrm{Hu}$ [15] concerning the role of nuclear spin networks in neural membranes in the emergence of consciousness through modulation of action potentials through J-coupling. In fact the flux density of the photons $\left(\sim 10^{-11} \mathrm{~W} \cdot \mathrm{m}^{-2}\right)$ measured from the right hemisphere of the cerebrum during periods when the subject imagined white light (vs mundane thoughts) was congruent with a diminishment of $\sim 5 \mathrm{nT}$ (within the range of the average fluctuation intensity of the quiet solar wind) as predicted by the dipole-dipole coupling relation across a classical neuronal plasma cell membrane.

If Vladimirsky and Bruns' [16] measurement variability of $5.2 \cdot 10^{-3}$ reported for $\mathrm{G}$ between days when the interplanetary magnetic field shifted from a positive to a negative sign and geomagnetic $A_{p}$ values ranged between -8 and $+8 \mathrm{nT}$ is applicable, then the role of subtle fluctuations in $G$ may be more direct than assumed. Persinger and St-Pierre [17], who completed lag/lead analyses on that data, found a Pearson $r$ coefficient of -0.50 and a Spearman rho $=-0.53$ (both, $\mathrm{p}<.05$ ). The negative correlation indicated that as $\mathrm{G}$ fluctuations decreased the perturbations associated with the local solar wind increased. In many contexts the inverse relationship strongly suggests a shared source of variance for both $G$ and the magnetic field associated with the solar interplanetary magnetic field.

The equivalence between magnetic field strength (B) and photon power density $\left(\mathrm{W} \cdot \mathrm{m}^{-2}\right)$ can be obtained by: 


$$
\left(\mathrm{kg} \cdot \mathrm{A}^{-1} \mathrm{~s}^{-2}\right) \cdot\left(\mathrm{A} \cdot \mathrm{s}^{-1}\right)=\mathrm{kg} \cdot \mathrm{s}^{3}
$$

For the two magnitudes to be equivalent the value for $\mathrm{A} \cdot \mathrm{s}^{-1}$ must be around $10^{-3} \mathrm{~A} \cdot \mathrm{s}^{-1}$ or in 1 $\mathrm{s}$, A. This quantity is well within the range of the capacity for the human cerebrum. Because a unit charge is $1.6 \cdot 10^{-19} \mathrm{~A} \cdot \mathrm{s}$, a summed value of $\sim 10^{-3} \mathrm{~A}$ there would be $\sim 10^{16}$ unit charges. Quantitative estimates indicate that the numbers of charges that maintain the passive potential of $\sim-70 \mathrm{mV}$ for the plasma membrane of a neuron is $\sim 10^{6}[5]$.

This means that per second there would be involvement of $10^{10}$ neurons or combinations of neurons and glial cells. Given that the time constant for most axons is around $100 \mathrm{~ms}$, this means that only about $10 \%$ of the total population would be maximally discharging per second. The resulting number is within the order of magnitude of the numbers of neurons in the human cerebral cortices.

\section{RELATING THE BOHR FREQUENCY TO RECOIL PHOTON ENERGY}

Bohr calculated the magnitude of the quantum involved if one nucleus was removed from another. The associated frequency was obtained by:

$$
v_{r}=1.32 \omega_{\mathrm{o}} \sqrt{ } \mathrm{m} \cdot \mathrm{M}^{-1}
$$

where $\mathrm{m}$ is the mass of an electron, $\mathrm{M}$ is the mass of a proton, and $\dot{\omega}_{0}$, orbital frequency, is $6.2 \cdot 10^{15} \mathrm{~Hz}$. The wave length for the solution, $\lambda_{r}$ is $1.54 \mu \mathrm{m}$, the approximate width of a synapse.

The frequency of the recoil energy of a photon, in terms of dimensional analysis, is $\mathrm{f}=$ $\mathrm{E} \cdot \hbar^{-1}$ where $\mathrm{E}$ is energy and $\hbar$ (the modified Planck's constant) is $1.06 \cdot 10^{-34} \mathrm{~J} \cdot \mathrm{s}$. If the equation is solved for $\mathrm{E}$, that is $1.32 \cdot \omega_{0}\left(\sqrt{\mathrm{m}} \cdot \mathrm{M}^{-1}\right) \cdot \hbar$, the energy is $2.15 \cdot 10^{-20} \mathrm{~J}$. This amount is equivalent to the quantum of energy associated with the effects of the change in voltage associated with an action potential upon a unit charge as well as the energy involved. In other words the recoil frequency of an atom when removed from another is equal to the fundamental quantum unit involved with multiple physicochemical and cognitive processes.

The recoil energy of an atom has also been described as:

$$
\mathrm{E}=\left[4 \mathrm{~h}^{2} \cdot \mathrm{k}^{2}\right] \cdot(2 \mathrm{~m})^{-1}
$$

where $\mathrm{k}$ is the wave number of the light, that is, the reciprocal of its wavelength, $\lambda^{-1}$, multiplied by $2 \pi$. If we differentiate $\mathrm{k}^{2}$ to $\lambda^{-1}$ and $1^{-1}$ where $\lambda$ is a mid-visible wavelength, in this instance $550 \mathrm{~nm}$, and 1 is the length of the physical dipole defined as the cerebrum (rostral-caudal distance), a potentially revealing amount of energy emerges if we assume the upper limit of the rest mass of a photon, $\sim 10^{-52} \mathrm{~kg}[18]$.

The calculation is: $\left(4.22 \cdot 10^{-68}\right) \cdot\left(1.81 \cdot 10^{7}\right) \cdot\left(10^{1}\right)$ divided by $2 \cdot 10^{-52}$, or, about $4 \cdot 10^{-9} \mathrm{~J}$. This is the same range of energy associated with the unit quantum of $2 \cdot 10^{-20} \mathrm{~J}$ per action potential for 20 billion cortical neurons discharging around $10 \mathrm{~Hz}$. This convergence suggests that thinking and cognition energy may be related to the recoil energy of a photon from its rest mass or its oscillation around rest mass. 


\section{SIGNIFICANT IMPLICATIONS FOR ASTRONOMY, PHYSICS AND CHEMISTRY}

There are three conspicuous implications of this solution. First, the recoil energy of a single photon from it rest mass within cerebral space would be equivalent to the sum of the neuroelectromagnetic correlates of cognition. This may be considered a characteristic of a hologram. Such conditions where $\mathrm{n}=\sum \mathrm{n}$ are also found within electrical potentials.

For example, dimensional analysis predicts:

$$
E=\sqrt{ }\left(V^{2} \cdot B \cdot C^{2}\right)
$$

where $\mathrm{B}$ equals the band width of the electroencephalographic activity, $\mathrm{V}$ is average voltage, and $\mathrm{C}$ is the unit charge. Assuming a typical value of $10 \mu \mathrm{V}^{2} \cdot \mathrm{Hz}^{-1}$, a $500 \mathrm{~Hz}$ range (to accommodate fast transients) and $2.56 \cdot 10^{-38} \mathrm{~A}^{2} \mathrm{~s}^{2}$ (square of the unit charge), the solution is $\sim 10^{-20} \mathrm{~J}$. Hence, the characteristics of the whole are reflected by the composite unit.

The second implication is that the recoil energy from a photon moving from its rest mass would be sufficient to simulate the entire cerebral cortical manifold and potentially to insert information that would constitute the equivalents of the energy typically associated with the activity of the billions of neurons whose field contributes to cognition and consciousness. There is experimental support that a single neuron's activity can affect the global state of the cerebrum [19].

However the recoil photon energy would be sufficient to both affect the state and potentially produce the states, at least briefly, from extracerebral sources. This possibility was first suggested by Sir Arthur Eddington [20] for which he received undeserved derision by his contemporaries. He appreciated that the perceptions and conceptions of information were actually representations within ensembles of neuronal activity and that an observation was a relation or a dynamic between two entities or particles. One of the implications is that the photons from "the universe" obtained by direct observation and those from cerebral processes could mutually affect each other.

That consciousness and cognition can be viewed as a field of photons as well as the more traditional neuronal ensembles was theorized by Bokkon [21] and has received significant theoretical and empirical support [22,23]. If photons are the primary sources of information upon which cells interact and from which perception and consciousness emerge, then the importance of "excess correlation", "non-locality" and "entanglement" becomes a quintessential component for the imminent shift in the scientific paradigm.

As discussed by Hofmann et al [24] "observers of two or more entangled particles will find correlations in their measurements that cannot be explained by classical statistics". Such excess correlation in photon emissions has been produced experimentally between pairs of brains separated by non-traditional distances that share specific characteristics of circular magnetic fields with changing angular accelerations whose phase and group velocities are dissociated [25].

Third, if the photon density and aggregate of quantum nodes within the cerebral volume is the functional dynamic of the human brain and its complex correlated activities such as perception, cognition, and consciousness, then the sources of information and determinant functions may be markedly different than assumed. The shared latency of $\sim 10^{-16} \mathrm{~s}$ for the passage of a photon moving at $\mathrm{c}$ across a plasma cell membrane and the time for an electron to complete one orbit could reflect a potential physical interface for exchange of information 
between the multiple orders of magnitude of space within and outside the volume that defines the brain.

The gravitational potential energy from the human brain can be estimated by:

$$
\mathrm{E}=\mathrm{G} \cdot \mathrm{kg}^{2} \cdot \mathrm{m}^{-1}
$$

Hence $\mathrm{G}=\left(6.67 \cdot 10^{-11} \mathrm{~m}^{3} \mathrm{~kg}^{-1} \mathrm{~s}^{-2}\right) \cdot(1.35 \mathrm{~kg})^{2} \cdot 1.2 \cdot 10^{-1} \mathrm{~m}^{-1}$ (average cerebral mass and length) results in $1.01 \cdot 10^{-9} \mathrm{~J}$. This is equivalent to the energy associated with approximately $10^{11}$ units of $10^{-20} \mathrm{~J}$ generated by each action potential [5] or about 10 billion neurons each firing at 10 $\mathrm{Hz}$. In other words the potential gravitational energy within the human cerebrum is congruent with the energy generated by the organic processes of neuronal function.

Because energies of the same magnitude are considered identities, there is the possibility that the gravitational and the neuroelectromagnetic sources could converge or even be substitutable. If cognition and consciousness are products of or strongly correlated with these energetic configurations the person engaging in the experience would not be able to discriminate the source. Experiences and memories could therefore originate from intracerebral neuronal-based processes, such as patterns of action potentials, or from the subtle intricacies of the gravitational energy within the cerebral mass.

If the fastest of the free oscillations of the earth, $3.7 \mathrm{mHz}$ [26], is considered the power is $3.7 \cdot 10^{-12} \mathrm{~W}$. When this value is divided by the actual cerebral cortical surface area of the human cerebrum $\left(1.82 \cdot 10^{-1} \mathrm{~m}^{2}\right)$ the power density is $2 \cdot 10^{-11} \mathrm{~W} \cdot \mathrm{m}^{-2}$ which is precisely the quantities involved with the photon emissions experimentally measured from the human cerebrum during imagination and thinking. If the suggested interface between electromagnetic quanta and gravitational energy occurs around the wavelength associated with visible light [27], then the quantitative similarity between photon irradiance and gravitational energy potential within the human cerebrum would be expected.

The relationship between the gravitational and electromagnetic energies could be validated experimentally. The numbers of charges moving per unit time within the volume of the cerebrum would be relatively fixed by its constant volume. The width of the range would be determined by the bulk frequency of the cerebral cortical activity. As a result the major variable that would affect the photon emissions would be the strength of the applied magnetic field.

Dotta et al [28] applied the concept of the Bohr magnetic moment to the passive diffusion of proteins and lipids within the plasma membrane. This "membrane magnetic moment" precisely predicted the quantitative peaks and shifts in biophoton emissions from aggregates of about 1 million (cancer) cells as a function of the intensity of the applied magnetic field. The maximum effect was measured around $1 \mu \mathrm{T}$.

However the role of gravitational energies might be included if the dimensional solution for:

$$
\mathrm{B} \cdot \mathrm{m}^{-1} \cdot \varphi \cdot \mathrm{v}^{-1} \mathrm{~A}=\mathrm{a}
$$

where $B$ is the magnetic field strength in Tesla $\left(\mathrm{kg} \cdot \mathrm{A}^{-1} \mathrm{~s}^{-2}\right), \mathrm{m}$ is the mass of the cerebrum, $\varphi$ is $\mathrm{A} \cdot \mathrm{s}^{-1}, \mathrm{v}=$ velocity, $\mathrm{A}=$ area and $\mathrm{a}=$ acceleration, is valid. Assuming $10^{-7} \mathrm{~T}(1 \mathrm{mG})$, a typical strength of the field strengths applied across the cerebrum in our research [29], cerebral mass of $1.35 \mathrm{~kg}$ [30], current temporal density of $10^{-3} \mathrm{~A} \cdot \mathrm{s}^{-1}$, a bulk velocity of $4.5 \mathrm{~m} \cdot \mathrm{s}^{-1}$ along the rostral-caudal axis of the cerebrum, and an area of $1.83 \cdot 10^{-1} \mathrm{~m}^{2}$ [30] the acceleration would be $\sim 0.5 \cdot 10^{-11} \mathrm{~m} \cdot \mathrm{s}^{-2}$. 
This is exactly within the range of the natural free oscillations of the earth [26]. The obvious prediction is that the experimental application of appropriately patterned 0.1 to $1 \mu \mathrm{T}$ magnetic fields to produce the intrinsic acceleration within the cerebrum that is coupled with the gravitational energy from equation (6) should be associated with a systematic increase in the spontaneous photon emission densities [23] from the cerebrum.

\section{CONCLUSIONS}

The duality of mass and energy requires that the fundamental interface, the synapse, for information within brain space should also be manifested as punctate units or quanta of energy in the order of $10^{-11} \mathrm{~W} \cdot \mathrm{m}^{-2}$ that can be measured by photomultiplier tubes. The potential equivalence of the same numbers of quanta within the cerebrum as the numbers synapses indicates that perception, cognition, and consciousness may actually be fields of photons that can be influenced by the brain's gravitational energy as well as very weak fluctuations in geomagnetic field intensities. Because the recoil energy of a single photon from its rest mass is equivalent to the energy associated with the summed action potentials of billions of neurons within the cerebrum, a single photon from this origin could simulate or replace the experiences normally associated with organic activity.

\section{References}

[1] A. D. Aczel, Entanglement: The Greatest Mystery in Physics Raincoast Books, Vancouver, 2002.

[2] H. Hu, M. Wu, NeuroQuantology 1 (2006) 5-16.

[3] K. H. Pribram, S. D. Meade, New Ideas in Psychology 17 (1999) 205-214.

[4] W. Ramsay, F. G. Donnan, Textbooks of Physical Chemistry: A System of Physical Chemistry, Longmans, Green and Co: London, 1921.

[5] M. A. Persinger, Current Medicinal Chemistry 17 (2010) 3094-3098.

[6] L.Y. Wei, Bulletin of Mathematical Biophysics 31 (1969) 39-58.

[7] G. M. Shepherd, The Synaptic Organization of the Brain Oxford University Press, Oxford, 2004.

[8] B. T. Dotta, C. A. Buckner, D. Cameron, R. M. Lafrenie, M. A. Persinger, General Physiology and Biophysics 30 (2011) 301-309.

[9] B. T. Dotta, C. A. Buckner, R. M. Lafrenie, M. A. Persinger, Brain Research 388 (2011) 77-88.

[10] B. T. Dotta, K. S. Saroka, M. A. Persinger, Neuroscience Letters 513 (2012) 151-154.

[11] M. D. Hunter, B. P. Mulligan, B. T. Dotta, K. S. Saroka, C. F. Lavalle, S. A. Koren, M. A. Persinger, Journal of Consciousness Exploration and Research 1 (2010) 1179-1197.

[12] D. A. E. Vares, M. A. Persinger, Journal of Nonlocality 2(2) (2013) 1-17.

[13] K. S. Saroka, B. T. Dotta, M. A. Persinger, International Journal of Life Science and Medical Research 3 (2013) 30-34. 
[14] M. A. Persinger, B. T. Dotta, K. S. Saroka, M. A. Scott, Journal of Consciousness Exploration \& Research 4 (2013) 1-24.

[15] H. Hu, M. Wu, NeuroQuantology 4 (2004) 309-317.

[16] B. M. Vladimirsky, A. V. Bruns, Biophysics 43 (1998) 720-725.

[17] M. A. Persinger, L. S. St-Pierre, International Journal of Geosciences (in submission)

[18] L. C. Tu, J. Luo, G. T. Gilles, Reports of Progress in Physics 68 (2005) 77-130.

[19] C. T. Li, M. Poo, Y. Dan, Science 324 (2009) 643-645.

[20] A. Eddington, The Nature of the Physical World University Michigan Press, Ann Arbor, 1958.

[21] I. Bokkon, Sleep and Hypnosis 7 (2005) 61-76.

[22] I. Bokkon, BioSystems 96 (2009) 178-184.

[23] I. Bokkon, V. Salari, J. A. Tuszynski, I. Antal, Journal of Photochemistry and Photobiology B, 100 (2010) 160-166.

[24] J. Hofmann, M. Krug, N. Ortegel, L. Gerard, M. Weber, W. Rosenfeld, H. Weinfurter, Science 337 (2012) 72-76.

[25] B. T. Dotta, M. A. Persinger, Journal of Biophysical Chemistry 3 (2012) 72-80.

[26] K. Nishida, N. Kobayashi, Y. Fukao, Science 287 (2000) 2244-2246.

[27] M. A. Persinger, The Open Astronomy Journal 5 (2012) 41-43.

[28] B. T. Dotta, R. M. Lafrenie, L. M. Karbowski, M. A. Persinger, General Physiology and Biophysics 33 (2014) 63-73.

[29] M. A. Persinger, K. S. Saroka, S. A. Koren, L. S. St-Pierre, Journal of Consciousness Exploration \& Research 1 (2010) 808-830.

[30] B. Pakkenberg, H. J. G. Gundersen, Journal of Comparative Neurology 384 (1997) 312-320. 